\title{
Stabilising systems with aperiodic sample-and-hold devices: state feedback case
}

\author{
$\operatorname{AUTHOR}(\mathrm{S})$ : \\ Fujioka, H.; Nakai, T.
}

\section{CITATION:}

Fujioka, H. ... [et al]. Stabilising systems with aperiodic sample-and-hold devices: state feedback case. IET Control Theory \& Applications 2010, 4(2): 265-272

\section{ISSUE DATE:}

2010-02

URL:

http://hdl.handle.net/2433/126693

\section{RIGHT:}

(c) 2010 Institution of Engineering and Technology; この論文は出版社版 でありません。引用の際には出版社版をご確認ご利用ください。; This is not the published version. Please cite only the published version. 


\title{
Stabilizing Systems with
}

\section{Aperiodic Sample-and-Hold Devices: State Feedback Case}

\author{
Hisaya Fujioka Toshiharu Nakai \\ Graduate School of Informatics, Kyoto University, Kyoto 606-8501, \\ Japan, email: \{fujioka, nakai\}@acs.i.kyoto-u.ac.jp
}

\begin{abstract}
Motivated by the widespread use of networked and/or embedded control systems, an algorithm for stabilizing sampled-data feedback control systems with uncertainly time-varying sampling intervals is proposed. The algorithm is an extension of that for stability analysis in the authors' previous study, and is based on the robustness against the variation of sampling intervals derived by the small-gain condition. The validity of the algorithm is demonstrated by numerical examples.
\end{abstract}

\section{Introduction}

The sampled-data control theory (See [2] and references therein) has been welldeveloped in the last two decades. One of the crucial properties in the development of the sampled-data control theory is the periodicity of the closed-loop systems which comes from periodic sampling.

We, however, recently encounter applications where periodic sampling is impossible. Networked and embedded control systems are typical examples, where resources for measurement and control are restricted (See $[8,9]$ and references therein) and hence the sampling operation tends to be aperiodic and uncertain. This fact motivates the study of systems with aperiodic sampling, where ro- 
bustness against variation of sampling intervals plays a crucial role since the sampling intervals are uncertain.

In the literature, both continuous and discrete-time models of the systems have been used for the study. In the continuous-time approach $[3,4,6,13$ 15, 18, 20-22] analysis and synthesis problems have been studied by using the methodologies developed for time-delay systems [3,4,14,18], hybrid systems [15], and robust control $[6,13]$. In the discrete-time approach, the state-space matrices of an exact discrete-time model depend on the time-varying sampling intervals in a nonlinear form. In order to avoid the difficulty, the model has been approximated by ignoring the higher order terms of the sampling intervals in the state-space matrices [1], or by replacing the set of possible sampling intervals by a set of a finite number of sampling intervals [16,21]. The approximations make, for example, the stability analysis problem tractable, however, the stability of the original system cannot be concluded. The discrete-time approach has also been taken in the recent reference [5] for the stability analysis, where the set of finite number of sampling intervals have been used similarly. However, the method in [5] can guarantee the stability of the original system by exploiting the robustness against the variation of sampling intervals based on the small-gain condition. Moreover, the proposed method provides less conservative results for the numerical examples in [5] compared to the continuous-time approach $[3,4,6,13-15,18,20-22]$.

The purpose of the present paper is to apply the method in [5] to a synthesis problem. In particular, we will provide a synthesis algorithm of constant feedback gain matrix which exponentially stabilizes the resulting system, under the condition that the sampled state is available for feedback.

This paper is a full and extended version of the conference paper [7]. After the conference presentation closely related results have been reported in [17]. One of the main advantages of the present paper compared to [17] is the development of a concrete algorithm for the state feedback synthesis with a guarantee of convergence, as we will see below.

This paper is organized as follows: The problem is formulated in Section 2. Section 3 provides a synthesis algorithm to exponentially stabilize resulting systems. The validity of the algorithm is demonstrated in Section 4 by numerical 


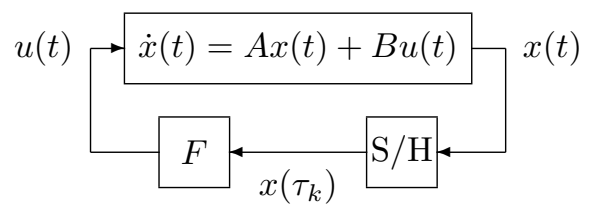

Figure 1: Feedback control with aperiodic sample-and-hold circuits

examples.

\section{Problem Formulation}

Let the following state-space system be given

$$
\dot{x}(t)=A x(t)+B u(t)
$$

where $x$ and $u$ respectively denote the state and the input taking values in $\mathbb{R}^{n}$ and $\mathbb{R}^{m}$. $A$ and $B$ are matrices of compatible dimensions.

We consider the following scenario of the feedback control of (1):

- We can measure the state of $(1)$ when $t=\tau_{k}(k=0,1, \ldots)$ where $\left\{\tau_{k}\right\}$ is an uncertain set of discrete time instants satisfying $\tau_{0}=0$ and

$$
0<h_{\ell} \leq \tau_{k+1}-\tau_{k} \leq h_{u}<\infty
$$

for given $h_{\ell}$ and $h_{u}$.

- The control input $u$ is determined by the sampled state $x\left(\tau_{k}\right)$ and a constant feedback gain $F \in \mathbb{R}^{m \times n}$, which is the design parameter in the present paper, with the zero-th order hold synchronizing the sampling:

$$
u(t)=F x\left(\tau_{k}\right), \quad{ }^{\forall} t \in\left[\tau_{k}, \tau_{k+1}\right) .
$$

Remark 1 The condition $h_{\ell}>0$ implies

$$
\lim _{k \rightarrow \infty} \tau_{k}=\infty
$$

The resulting feedback system composed of (1) and (3), denoted by $T$, is given by

$$
\dot{x}(t)=A x(t)+B F x\left(\tau_{k}\right), \quad{ }^{\forall} t \in\left[\tau_{k}, \tau_{k+1}\right) .
$$


See also Fig. 1. Applications of this scenario can be found in networked and/or embedded control systems $[8,9]$, where resources for measurement and control are restricted.

The purpose of the present paper is to provide a synthesis algorithm of $F$ for stabilizing $T$. If $\tau_{k}$ 's satisfy

$$
\tau_{k+1}-\tau_{k}=h
$$

for some $h \in\left[h_{\ell}, h_{u}\right]$, the resulting feedback control system $T$ is periodic. This special scenario is the one well-studied in the so-called sampled-data control theory [2]. Indeed the stability can be easily verified by checking the spectral radius of $\Phi(h)$ in the special scenario, where

$$
\begin{gathered}
\Phi_{F}(h):=\Phi(h)+\Gamma(h) F, \\
\Phi(h):=\mathrm{e}^{A h}, \quad \Gamma(h):=\int_{0}^{h} \mathrm{e}^{A(h-\eta)} B \mathrm{~d} \eta,
\end{gathered}
$$

and finding a stabilizing $F$ is routine. It is, however, readily seen that our general scenario is much more complicated, because of the uncertain time-varying nature.

In the present paper we will develop a stabilization algorithm based on the following lemma $[8,21]$ :

Lemma $1 T$ is exponentially stable if there exists a matrix $0<P=P^{\top} \in \mathbb{R}^{n \times n}$ satisfying

$$
\left(\Phi_{F}(h)\right)^{\top} P \Phi_{F}(h)-P<0
$$

for all $h \in\left[h_{\ell}, h_{u}\right]$, where $\Phi_{F}(\cdot)$ is defined in (6).

Note that Lemma 1 is based on the quadratic stability of the accompanying discrete-time system $T_{d}$ defined by

$$
\xi[k+1]=\Phi_{F}\left(\tau_{k+1}-\tau_{k}\right) \xi[k]
$$

with the discrete-time Lyapunov function

$$
V(\xi[k]):=\xi^{\top}[k] P \xi[k]
$$

where $\xi[k]:=x\left(\tau_{k}\right)$. 
Note also that it is hard to find a matrix $P$ in Lemma 1 since the inequality must hold for all values in $\left[h_{\ell}, h_{u}\right]$. In the previous study [5] we have developed a stability analysis algorithm based on Lemma 1 for a given $F$. In the present paper our purpose is to find $P$ and $F$ simultaneously.

\section{Main Results}

In the previous study of [5] we have developed an algorithm to construct a finite grid:

$$
\mathcal{G}=\left\{h_{1}, h_{2}, \ldots, h_{N}\right\} \subset\left[h_{\ell}, h_{u}\right]
$$

so that the existence of $P>0$ satisfying (7) for all $h \in \mathcal{G}$ guarantees the existence of $P>0$ satisfying (7) for all $h \in\left[h_{\ell}, h_{u}\right]$. In other words, we have provided an estimate of the robustness of systems with uniform sampling interval against the perturbation caused by the variation of sampling interval. In this section this idea will be applied to the synthesis problem.

\subsection{Robust Stabilization against Variation of Sampling In- tervals}

In order to discuss the robustness against the variation of sampling interval, we consider the following manipulation of $\Phi_{F}$ : Fixing $h_{0} \in\left(h_{\ell}, h_{u}\right)$ one can define $\theta_{k}$ so that

$$
\tau_{k+1}-\tau_{k}=h_{0}+\theta_{k}
$$

One has the following property found in [5], which is simple but plays a key role in the present paper:

Proposition 1 The function $\Phi_{F}(\cdot)$ defined in (6) satisfies

$$
\Phi_{F}\left(\tau_{k+1}-\tau_{k}\right)=\Phi_{F}\left(h_{0}\right)+\Delta\left(\theta_{k}\right) \Psi_{F}\left(h_{0}\right)
$$

where

$$
\begin{gathered}
\Psi_{F}(h):=\Psi(h)+\Upsilon(h) F, \\
\Psi(h):=A \Phi(h), \quad \Upsilon(h):=A \Gamma(h)+B, \\
\Delta(\theta):=\int_{0}^{\theta} \mathrm{e}^{A \eta} \mathrm{d} \eta .
\end{gathered}
$$




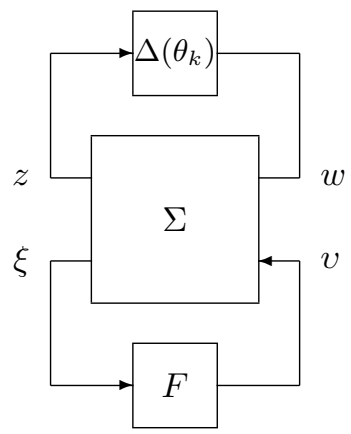

Figure 2: Alternative representation of $T_{d}$

Proof By definition

$$
\Phi_{F}\left(\tau_{k+1}-\tau_{k}\right)=\Phi\left(h_{0}+\theta_{k}\right)+\Gamma\left(h_{0}+\theta_{k}\right) F
$$

The first term of the right hand side can be transformed to

$$
\Phi\left(h_{0}+\theta_{k}\right)=\mathrm{e}^{A \theta_{k}} \Phi\left(h_{0}\right)=\left(I+\Delta\left(\theta_{k}\right) A\right) \Phi\left(h_{0}\right) .
$$

On the other hand for the second term we have the following:

$$
\begin{aligned}
& \Gamma\left(h_{0}+\theta_{k}\right) \\
= & \int_{0}^{h_{0}} \mathrm{e}^{A\left(h_{0}+\theta_{k}-\eta\right)} B \mathrm{~d} \eta+\int_{h_{0}}^{h_{0}+\theta_{k}} \mathrm{e}^{A\left(h_{0}+\theta_{k}-\eta\right)} B \mathrm{~d} \eta \\
= & \mathrm{e}^{A \theta_{k}} \Gamma\left(h_{0}\right)+\Delta\left(\theta_{k}\right) B \\
= & \left(I+\Delta\left(\theta_{k}\right) A\right) \Gamma\left(h_{0}\right)+\Delta\left(\theta_{k}\right) B .
\end{aligned}
$$

Then it is straightforward to derive (8) by substituting the above results to the right hand side of the first equation in this proof.

Now one can regard $T_{d}$ as a feedback connection of a time-varying matrix $\Delta\left(\theta_{k}\right)$ and an LTI discrete-time system $\mathcal{F}_{\ell}(\Sigma, F)$, where $\Sigma$ is given by

$$
\Sigma:\left[\begin{array}{c}
\xi[k+1] \\
z[k] \\
\xi[k]
\end{array}\right]=\left[\begin{array}{ccc}
\Phi\left(h_{0}\right) & I & \Gamma\left(h_{0}\right) \\
\Psi\left(h_{0}\right) & 0 & \Upsilon\left(h_{0}\right) \\
I & 0 & 0
\end{array}\right]\left[\begin{array}{c}
\xi[k] \\
w[k] \\
v[k]
\end{array}\right] .
$$

See Fig. 2. Thus we obtain the following lemma:

Lemma 2 Let an interval $\mathcal{H} \subseteq(0, \infty)$ be given. There exists a matrix $0<P=$ $P^{\top} \in \mathbb{R}^{n \times n}$ satisfying (7) for all $h \in \mathcal{H}$ if $\rho\left(\Phi_{F}\left(h_{0}\right)\right)<1$ and

$$
\gamma\|\Delta(\theta)\| \leq 1
$$


for all $\theta \in \mathcal{H}-h_{0}$, where $\gamma$ is a strict upper bound of $\left\|\mathcal{F}_{\ell}(\Sigma, F)\right\|_{\infty}$ :

$$
\gamma>\left\|\mathcal{F}_{\ell}(\Sigma, F)\right\|_{\infty}
$$

This is a consequence of the relationship between the quadratic stability and the small-gain condition discussed in, e.g., [12]. Here we provide a proof for completeness of the paper.

Proof Invoking the bounded real lemma (see, e.g., [23]), (11) implies that there exists a matrix $X=X^{\top}>0$ satisfying

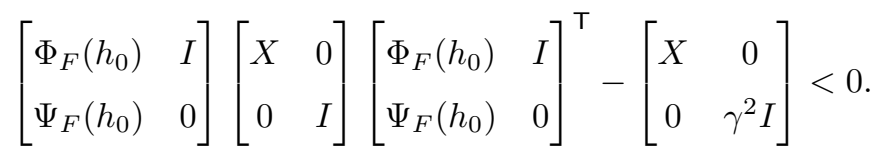

After manipulations using the Schur complement lemma, this turns to

$$
\left[\begin{array}{cc}
X^{-1} & -\Phi_{F}^{\top}\left(h_{0}\right) \\
-\Phi_{F}\left(h_{0}\right) & X
\end{array}\right]-\left[\begin{array}{cc}
\gamma^{-2} \Psi_{F}^{\top}\left(h_{0}\right) \Psi_{F}\left(h_{0}\right) & 0 \\
0 & I
\end{array}\right]>0
$$

Substituting $I>\gamma^{2} \Delta(\theta) \Delta^{\top}(\theta)$, which is implied from (10), to the above inequality we have

$$
\left[\begin{array}{cc}
X^{-1} & -\Phi_{F}^{\top}\left(h_{0}\right) \\
-\Phi_{F}\left(h_{0}\right) & X
\end{array}\right]-\left[\begin{array}{cc}
\gamma^{-2} \Psi_{F}^{\top}\left(h_{0}\right) \Psi_{F}\left(h_{0}\right) & 0 \\
0 & \gamma^{2} \Delta(\theta) \Delta^{\top}(\theta)
\end{array}\right]>0 .
$$

Noting the fact (see, e.g., [12])

$$
\left[\begin{array}{cc}
\gamma^{-2} \Psi_{F}^{\top}\left(h_{0}\right) \Psi_{F}\left(h_{0}\right) & 0 \\
0 & \gamma^{2} \Delta(\theta) \Delta^{\top}(\theta)
\end{array}\right] \geq\left[\begin{array}{c}
\Psi_{F}^{\top}\left(h_{0}\right) \\
0
\end{array}\right]\left[\begin{array}{ll}
0 & \Delta^{\top}(\theta)
\end{array}\right]+\left[\begin{array}{c}
0 \\
\Delta(\theta)
\end{array}\right]\left[\begin{array}{ll}
\Psi_{F}\left(h_{0}\right) & 0
\end{array}\right]
$$

we finally obtain

$$
\left[\begin{array}{cc}
X^{-1} & -\Phi_{F}^{\top}\left(h_{0}\right)-\Psi_{F}^{\top}\left(h_{0}\right) \Delta^{\top}(\theta) \\
-\Phi_{F}\left(h_{0}\right)-\Delta(\theta) \Psi_{F}\left(h_{0}\right) & X
\end{array}\right]>0
$$

which turns to

$$
\left(\Phi_{F}\left(h_{0}\right)+\Delta(\theta) \Psi_{F}\left(h_{0}\right)\right)^{\top} X^{-1}\left(\Phi_{F}\left(h_{0}\right)+\Delta(\theta) \Psi_{F}\left(h_{0}\right)\right)-X^{-1}<0 .
$$

By letting $P=X^{-1}$ the proof is complete. 
Since (stabilization of $\mathcal{F}_{\ell}(\Sigma, F)$ and) minimization of $\gamma$ in (11) is routine, one can stabilize $T$ based on (10) by bounding $\|\Delta(\theta)\|$. For the purpose we invoke the following property in, e.g., [19]:

Lemma 3 For given $A \in \mathbb{R}^{n \times n}$ and $t \geq 0$ one has

$$
\left\|\mathrm{e}^{A t}\right\| \leq \mathrm{e}^{\mu(A) t}
$$

where $\mu(A)$ denotes the log norm of $A$ :

$$
\mu(A)=\lambda_{\max }\left(\frac{A+A^{\top}}{2}\right) .
$$

Remark 2 One can continue the following discussion by replacing the bound in (12) by other bounds found in, e.g., [10,19].

Hence we have the following basic robustness results, which is an alternative representation of Theorem 1 in [5]:

Theorem 1 Let $h_{0}>0, F$, and $\gamma>0$ be given so that $\rho\left(\Phi_{F}\left(h_{0}\right)\right)<1$ and (11) holds. There exists a matrix $0<P=P^{\top} \in \mathbb{R}^{n \times n}$ satisfying (7) for all $h \in \mathcal{H}\left(h_{0}, \gamma\right)$, where

$$
\mathcal{H}\left(h_{0}, \gamma\right):=\left[h_{L}, h_{U}\right] \cap(0, \infty),
$$

and $h_{L}$ and $h_{U}$ are given as follows:

L1) If $\mu(-A)=0, h_{L}=h_{0}-\gamma^{-1}$,

L2) else if $\mu(-A) \leq-\gamma, h_{L}=-\infty$,

L3) else

$$
h_{L}=h_{0}-\frac{1}{\mu(-A)} \log \left(1+\gamma^{-1} \mu(-A)\right) \text {. }
$$

U1) If $\mu(A)=0, h_{U}=h_{0}+\gamma^{-1}$,

U2) else if $\mu(A) \leq-\gamma, h_{U}=\infty$,

U3) else

$$
h_{U}=h_{0}+\frac{1}{\mu(A)} \log \left(1+\gamma^{-1} \mu(A)\right) \text {. }
$$

Proof See Appendix. 


\subsection{Algorithm for State Feedback Synthesis}

Theorem 1 provides a robustness condition for $T$ based on the property of the nominal system determined by the fixed sampling period $h_{0}$. Hence it is straightforward to apply Theorem 1 to the state feedback synthesis problem, i.e., the stabilization problem of $T$ is cast into an $\mathbf{H}_{\infty}$ control problem for $\Sigma$ with an appropriate $h_{0}>0$, and the $\mathbf{H}_{\infty}$ control gain $F$ stabilizes $T$ provided that $\left[h_{\ell}, h_{u}\right] \subseteq \mathcal{H}\left(h_{0}, \gamma\right)$ is satisfied. This direct use of Theorem 1 , however, can be conservative in the sense that there might not exist $h_{0}>0$ and $F$ such that $\left[h_{\ell}, h_{u}\right] \subseteq \mathcal{H}\left(h_{0}, \gamma\right)$ even though there exists a matrix $P$ satisfying (7) for all $h \in\left[h_{\ell}, h_{u}\right]$, mainly because of the small-gain type modeling of $\Delta\left(\theta_{k}\right)$.

In order to reduce the conservatism we introduce the method similar to the one in the multi-objective LMI synthesis (see, e.g., [11]) to obtain the following theorem:

Theorem 2 Let $h_{i}>0(i=1,2, \ldots, N)$ be given. If there exist a matrix $0<X=X^{\top} \in \mathbb{R}^{n \times n}, W \in \mathbb{R}^{m \times n}$, and $\alpha_{i}>0(i=1,2, \ldots, N)$ satisfying the following $N$ linear matrix inequalities

$$
\left[\begin{array}{ccc}
X-I & 0 & L_{1}\left(h_{i}, X, W\right) \\
0 & \alpha_{i} I & L_{2}\left(h_{i}, X, W\right) \\
L_{1}^{\top}\left(h_{i}, X, W\right) & L_{2}^{\top}\left(h_{i}, X, W\right) & X
\end{array}\right]>0
$$

then (7) is satisfied with $P=X^{-1}, F=W X^{-1}$ for all

$$
h \in \bigcup_{i=1}^{N} \mathcal{H}\left(h_{i}, \sqrt{\alpha_{i}}\right)
$$

where $\Phi(\cdot), \Gamma(\cdot), \Psi(\cdot), \Upsilon(\cdot), \mathcal{H}(\cdot, \cdot)$ are defined in (6), (9), and (13), respectively, and

$$
\begin{aligned}
& L_{1}\left(h_{i}, X, W\right):=\Phi\left(h_{i}\right) X+\Gamma\left(h_{i}\right) W, \\
& L_{2}\left(h_{i}, X, W\right):=\Psi\left(h_{i}\right) X+\Upsilon\left(h_{i}\right) W .
\end{aligned}
$$

Proof Consider the case $i=1$. Invoking the Shur complement lemma, we see that (14) for $i=1$ is equivalent to

$$
\left[\begin{array}{cc}
X-I & 0 \\
0 & \alpha_{1} I
\end{array}\right]-\left[\begin{array}{c}
L_{1}\left(h_{1}, X, W\right) \\
L_{2}\left(h_{1}, X, W\right)
\end{array}\right] X^{-1}\left[L_{1}^{\top}\left(h_{1}, X, W\right) \quad L_{2}^{\top}\left(h_{1}, X, W\right)\right]>0 .
$$


This turns to

$$
\left[\begin{array}{ll}
\Phi_{W X^{-1}}\left(h_{1}\right) & I \\
\Psi_{W X^{-1}}\left(h_{1}\right) & 0
\end{array}\right]\left[\begin{array}{cc}
X & 0 \\
0 & I
\end{array}\right]\left[\begin{array}{ll}
\Phi_{W X^{-1}}\left(h_{1}\right) & I \\
\Psi_{W X^{-1}}\left(h_{1}\right) & 0
\end{array}\right]^{\top}-\left[\begin{array}{cc}
X & 0 \\
0 & \alpha_{1} I
\end{array}\right]<0 .
$$

Invoking the bounded real lemma, this is equivalent to

$$
\left\|\mathcal{F}_{\ell}\left(\Sigma, W X^{-1}\right)\right\|_{\infty}<\sqrt{\alpha_{1}} .
$$

Hence, by Theorem 1 , there exists a matrix $0<P=P^{\top} \in \mathbb{R}^{n \times n}$ satisfying (7) for all $h \in \mathcal{H}\left(h_{1}, \sqrt{\alpha_{1}}\right)$ when $F=W X^{-1}$. Moreover one of such $P$ 's is given by $X^{-1}$ as shown in Proof of Lemma 2. In exactly the same way, we can conclude that there exists a matrix $0<P=P^{\top}=X^{-1} \in \mathbb{R}^{n \times n}$ satisfying (7) for all $h \in \mathcal{H}\left(h_{i}, \sqrt{\alpha_{i}}\right), i=2, \ldots, N$. This concludes the proof.

Remark 3 Since F must be shared by all the $h_{i}$ 's, one cannot apply the socalled variable elimination method in the reduction to LMIs.

Once a grid of sampling intervals is fixed by some method, one can invoke Theorem 2 to find a matrix $P>0$ and $F$ satisfying (7). Moreover, if there exists a pair of $P$ and $F$ satisfying (7), then there exists a grid verifying it.

Proposition 2 Suppose that there exists a pair of $P=P^{\top}>0$ and $F$ satisfying (7) for all $\left[h_{\ell}, h_{u}\right]$. There exists a finite set $\left\{h_{i}\right\}_{i=1}^{N}$ such that there exist $X=$ $X^{\top}>0, W$ and $\alpha_{i}$ 's satisfying (14) and

$$
\left[h_{\ell}, h_{u}\right] \subseteq \bigcup_{i=1}^{N} \mathcal{H}\left(h_{i}, \sqrt{\alpha_{i}}\right)
$$

Proof The existence of $P$ and $F$ implies that of $X=X^{\top}>0, W$, and $\epsilon>0$ satisfying

$$
\left[\begin{array}{ccc}
X-I & 0 & L_{1}(h, X, W) \\
0 & \left(1 / \epsilon^{2}\right) I & L_{2}(h, X, W) \\
L_{1}^{\top}(h, X, W) & L_{2}^{\top}(h, X, W) & X
\end{array}\right]>0
$$

for all $h \in\left[h_{\ell}, h_{u}\right]$. Hence there must exist a pair of $X$ and $W$ satisfying (14) when we take $\alpha_{i}=1 / \epsilon^{2}$ for all $i$ 's. Let us consider the case of $\mu(A)>-1 / \epsilon$ and $\mu(-A)>-1 / \epsilon$. Proofs for cases of $\mu(A) \leq-1 / \epsilon$ or $\mu(-A) \leq-1 / \epsilon$ are easier. 
Let $2 \eta$ denote the width of the interval $\mathcal{H}\left(h_{i}, 1 / \epsilon\right)$ which is independent of $h_{i}$ and determined by $\mu(A), \mu(-A)$, and $\epsilon$. Then (15) can be achieved by taking a grid

$$
\left\{h_{\ell}+\eta, h_{\ell}+3 \eta, h_{\ell}+5 \eta, \ldots, h_{\ell}+\left(2 N_{1}-1\right) \eta, \min \left(h_{\ell}+\left(2 N_{1}+1\right) \eta, h_{u}\right)\right\},
$$

where $N_{1}$ is the natural number so that

$$
h_{\ell}+2 N_{1} \eta<h_{u} \leq h_{\ell}+\left(2 N_{1}+2\right) \eta
$$

is satisfied.

In the present paper we propose the following concrete algorithm for stabilization which generates a grid effectively based on Theorem 2 . We denote the number of elements in a finite set $\mathcal{G}$ by $\#(\mathcal{G})$.

Algorithm 1 Given $0<h_{\ell}<h_{u}<\infty$, and a large positive integer $N_{0}$.

0. Initialization: $\mathcal{G} \leftarrow\left\{\left(h_{\ell}+h_{u}\right) / 2\right\}$

1. If there exists an $h_{0} \in \mathcal{G}$ such that $\left(\Phi\left(h_{0}\right), \Gamma\left(h_{0}\right)\right)$ is not stabilizable, there is no $F$ which stabilizes T. Stop.

2. If $\#(\mathcal{G}) \geq N_{0}$, stop without obtaining a stabilizing $F$.

3. Minimize

$$
\sum_{i=1}^{\#(\mathcal{G})} \beta_{i}
$$

subject to

$$
\left[\begin{array}{ccc}
X-I & 0 & L_{1}\left(h_{i}, X, W\right) \\
0 & \beta_{i} I & L_{2}\left(h_{i}, X, W\right) \\
L_{1}^{\top}\left(h_{i}, X, W\right) & L_{2}^{\top}\left(h_{i}, X, W\right) & X
\end{array}\right]>0
$$

for all $i$ 's over $0<X=X^{\top} \in \mathbb{R}^{n \times n}, W \in \mathbb{R}^{m \times n}$, and $\beta_{i} \geq 0$, where $h_{i}$ is the $i$-th smallest element in $\mathcal{G}$.

4. If

$$
\left[h_{\ell}, h_{u}\right] \subseteq \bigcup_{i=1}^{\#(\mathcal{G})} \mathcal{H}\left(h_{i}, \sqrt{\alpha_{i}}\right)
$$


$T$ is exponentially stabilized by $F:=W X^{-1}$. Stop. Here

$$
\alpha_{i}:=\lambda_{\max }\left(R_{i}-S_{i}^{\top}\left(Q_{i}-X\right)^{-1} S_{i}\right)+\varepsilon
$$

where $\varepsilon$ is a small positive number and

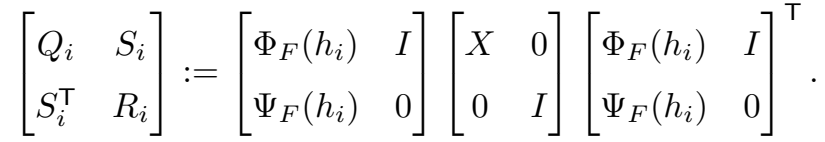

5. Update $\mathcal{G}$ by

$$
\mathcal{G} \leftarrow \mathcal{G} \cup\left\{\left(L_{j}+U_{j}\right) / 2\right\}
$$

for all $j$ where $L_{j}$ and $U_{j}$ are determined so that

$$
\begin{gathered}
\bigcup_{j=1}^{M}\left(L_{j}, U_{j}\right)=\left(h_{\ell}, h_{u}\right) \backslash \bigcup_{i=1}^{\#(\mathcal{G})} \mathcal{H}\left(h_{i}, \sqrt{\alpha_{i}}\right), \\
L_{1}<U_{1}<L_{2}<U_{2}<\cdots<L_{M}<U_{M}
\end{gathered}
$$

are satisfied. Go to Step 1.

We have some remarks for Algorithm 1:

- Step 2 is introduced to avoid numerical issues which could happen when $\#(\mathcal{G})$ is too large. Although Proposition 2 guarantees in theory that Algorithm 1 provides $F$ as long as $P$ and $F$ exist, LMI solvers may fail with a large number of LMIs to be solved simultaneously even if there exist a solution.

- The performance of the algorithm can be tuned by modifying the objective function in Step 3. Since $\beta_{i}$ is obtained so that the objective function is minimized, there may exist an $\alpha_{i} \leq \beta_{i}$ such that (14) holds with $X$ determined in Step 3. Note that $\mathcal{H}\left(h_{i}, \sqrt{\alpha_{i}}\right) \supseteq \mathcal{H}\left(h_{i} \sqrt{\beta_{i}}\right)$ when $\alpha_{i} \leq \beta_{i}$. Such $\alpha_{i}$ is obtained in Step 4 with sufficiently small $\varepsilon>0$, where $\varepsilon$ is taken for the strictness of the inequality (14).

- The integer $M$ in Step 5 is \# $(\mathcal{G})+1$ at most. 

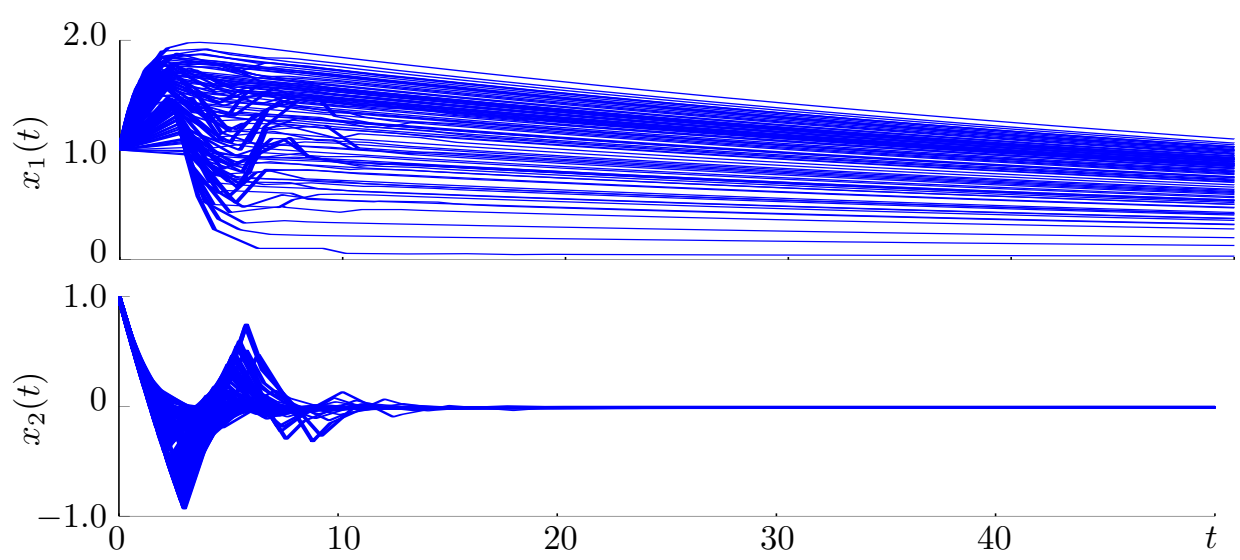

Figure 3: Initial value responses

Table 1: $\#(\mathcal{G})$ and CPU time varying $h_{u}$

\begin{tabular}{|c||c|c|c|c|c|c|}
\hline$h_{u}$ & 1.0 & 3.0 & 5.0 & 10.0 & 20.0 & 27.0 \\
\hline$\#(\mathcal{G})$ & 5 & 26 & 57 & 139 & 319 & 493 \\
CPU time $[\mathrm{s}]$ & 0.74 & 3.20 & 9.77 & 29.36 & 126.98 & 263.16 \\
\hline
\end{tabular}

\section{Numerical Examples}

In this section we demonstrate the validity of the proposed method for stability synthesis.

Let us consider the following problem parameters [21]:

$$
A=\left[\begin{array}{cc}
0 & 1 \\
0 & -0.1
\end{array}\right], \quad B=\left[\begin{array}{c}
0 \\
0.1
\end{array}\right]
$$

The proposed algorithm has provided a stabilizing gain

$$
F=-\left[\begin{array}{ll}
0.094 & 6.40
\end{array}\right]
$$

after 6 iterations with $\#(\mathcal{G})=26$ for $h_{\ell}=0.01$ and $h_{u}=3.0$, where we have used Robust Control Toolbox on MATLAB ${ }^{1}$ as an LMI solver. The CPU time for the computation was 3.20 seconds with Core2Duo $3.0 \mathrm{GHz}$ processor. Fig. 3 shows the 100 initial value responses with $x(0)=\left[\begin{array}{ll}1 & 1\end{array}\right]^{\top}$ where $\left\{\tau_{k}\right\}$ 's are randomly generated for $h_{\ell}=0.01$ and $h_{u}=3.0$.

For $N_{0}=500$ and $h_{\ell}=0.01$ we have found stabilizing $F$ for $h_{u} \leq 27.0$. Table 1 shows $\#(\mathcal{G})$ and the CPU time for each $h_{u}$. We can see the growth of

\footnotetext{
${ }^{1}$ http://www . mathworks.com/products/robust/
} 
the computational burden for larger $h_{u}$.

\section{Concluding Remarks}

We have considered the stabilization problem via state feedback for sampleddata feedback control systems where the state is sampled aperiodically, motivated by widespread use of networked and embedded control systems. We have developed a synthesis algorithm of a state feedback gain with which the resultant closed-loop system is exponentially stable, based on robustness of sampled-data systems against perturbation caused by variation of sampling intervals. The proposed algorithm is based on LMIs and the effectiveness has been verified by numerical examples.

There are several future directions: More practical synthesis problems including output feedback scenario should be considered as a direct extension of the present paper. We need to develop an alternative to Lemma 1 for the case where the exponential stability is too strong. It is also important to investigate analysis and synthesis problems with more specific performance of the closed-loop system such as an induced norm taking account of the intersample behavior.

\section{References}

[1] E. Boje. Approximate models for continuous-time linear systems with sampling jitter. Automatica, 41:2091-2098, 2005.

[2] T. Chen and B. A. Francis. Optimal Sampled-Data Control Systems. Springer, 1995.

[3] E. Fridman, A. Seuret, and J.-P. Richard. Robust sampled-data stabilization of linear systems: an input delay approach. Automatica, 40:1441-1446, 2004.

[4] E. Fridman, U. Shaked, and V. Suplin. Input/output delay approach to robust sampled-data $H_{\infty}$ control. Systems \&3 Control Letters, 54:271-282, 2005. 
[5] H. Fujioka. Stability analysis for a class of networked/embedded control systems: A discrete-time approach. In Proceedings of 2008 American Control Conference, pages 4997-5002, 2008.

[6] H. Fujioka. Stability analysis of systems with aperiodic sample-and-hold devices. Automatica, 45:771-775, 2009.

[7] H. Fujioka and T. Nakai. Stabilizing systems with aperiodic sample-andhold devices: state feedback case. In Proceedings of UKACC, 2008.

[8] J. P. Hespanha, P. Naghshtabrizi, and Y. Xu. A survey of recent results in networked control systems. Proceedings of the IEEE, 95:138-162, 2007.

[9] D. Hristu-Varsakelis and W. S. Levine, editors. Handbook of Networked and Embedded Control Systems. Birkhäuser, 2005.

[10] B. Kågström. Bounds and perturbation bounds for the matrix exponential. BIT, 17:89-97, 1977.

[11] I. Kaminer, P. P. Khargonekar, and M. Rotea. Mixed $\mathcal{H}_{2} / \mathcal{H}_{\infty}$ control for discrete-time systems via convex optimization. Automatica, 29:57-70, 1993.

[12] P. P. Khargonekar, I. R. Petersen, and K. Zhou. Robust stabilization of uncertain linear systems: Quadratic stabilizability and $H^{\infty}$ control theory. IEEE Transactions on Automatic Control, 35:356-361, 1990.

[13] L. Mirkin. Some remarks on the use of time-varying delay to model sampleand-hold circuits. IEEE Transactions on Automatic Control, 52:1109-1112, 2007.

[14] P. Naghshtabrizi and J. P. Hespanha. Designing an observer-based controller for a network control system. In Proceedings of the 44th IEEE Conference on Decision and Control, and the European Control Conference 2005, pages 848-853, 2005.

[15] P. Naghshtabrizi, J. P. Hespanha, and R. Teel. On the robust stability and stabilization of sampled-data systems: A hybrid system aproach. In Proceedings of the 45th IEEE Conference on Decision and Control, pages 4873-4878, 2006. 
[16] A. Sala. Computer control under time-varying sampling period: An LMI gridding approach. Automatica, 41:2077-2082, 2005.

[17] Y. S. Suh. Stability and stabilization of nonuniform sampling systems. Automatica, 44:3222-3226, 2008.

[18] V. Suplin, E. Fridman, and U. Shaked. Sampled-data $H_{\infty}$ control and filtering: Nonuniform uncertain sampling. Automatica, 43:1072-1083, 2007.

[19] C. Van Loan. The sensitivity of the matrix exponential. SIAM Journal of Numerical Analysis, 14:971-981, 1977.

[20] G. C. Walsh, H. Ye, and L. Bushnell. Stability analysis of networked control systems. In Proceedings of 1999 American Control Conference, pages 2876 2880, 1999.

[21] W. Zhang and M. S. Branicky. Stability of networked control systems with time-varying transmission period. In Allerton Conf. Communication, Contr. and Computing, 2001.

[22] W. Zhang, M. S. Branicky, and S. M. Phillips. Stability of networked control systems. IEEE Control Systems Maganine, 21:84-99, 2001.

[23] K. Zhou, J. C. Doyle, and K. Glover. Robust and Optimal Control. Prentice Hall, 1996.

\section{Proof of Theorem 1}

We here prove that $(10)$ holds for all $h \in\left[h_{0}, h_{U}\right]$. The proof for the interval $\left[h_{L}, h_{0}\right]$ is similar so it is omitted. Note that $\mathcal{H}\left(h_{0}, \gamma\right) \subseteq\left[h_{L}, h_{U}\right]$. The proof of the theorem then follows from the application of Lemma 2.

Invoking Lemma 3 we have

$$
\|\Delta(\theta)\| \leq \int_{0}^{\theta}\left\|\mathrm{e}^{A t}\right\| \mathrm{d} t \leq \int_{0}^{\theta} \mathrm{e}^{\mu(A) t} \mathrm{~d} t
$$

when $\theta \geq 0$. If $\mu(A)=0$

$$
\|\Delta(\theta)\| \leq \theta
$$

Hence (10) holds as long as $\gamma \theta \leq 1$. This completes the proof for the case U1. 
Let us next consider the case of $\mu(A) \neq 0$. In this case

$$
\|\Delta(\theta)\| \leq \frac{\mathrm{e}^{\mu(A) \theta}-1}{\mu(A)} .
$$

Suppose that $\mu(A)<0$. Note that the right hand side goes to $-1 / \mu(A)$ when $\theta$ tends to $\infty$. Hence (10) holds for all $\theta>0$ if

$$
-\frac{\gamma}{\mu(A)} \leq 1
$$

This completes the proof for the case U2.

Finally let us consider the case of $\mu(A) \neq 0$ and

$$
-\frac{\gamma}{\mu(A)}>1
$$

Invoking the small gain condition (see, e.g., [23]), (10) holds for all $\theta>0$ if

$$
\gamma \frac{\mathrm{e}^{\mu(A) \theta}-1}{\mu(A)} \leq 1 .
$$

Noting that $1+\gamma^{-1} \mu(A)>0$ in this case, this condition turns to

Case A) If $\mu(A)>0$

$$
\mu(A) \theta \leq \log \left(1+\gamma^{-1} \mu(A)\right)
$$

Case B) If $\mu(A)<0$

$$
\mu(A) \theta \geq \log \left(1+\gamma^{-1} \mu(A)\right)
$$

Hence we have

$$
\theta \geq \frac{1}{\mu(A)} \log \left(1+\gamma^{-1} \mu(A)\right)
$$

for both cases. This completes the proof for the case U3. 